\title{
MabA (FabG1), a Mycobacterium tuberculosis protein involved in the long-chain fatty acid elongation system FAS-II
}

\author{
Hedia Marrakchi, ${ }^{1} \dagger \neq$ Stéphanie Ducasse, ${ }^{1} \ddagger$ Gilles Labesse, ${ }^{2}$ \\ Henri Montrozier, ${ }^{1}$ Emmanuel Margeat, ${ }^{2}$ Laurent Emorine, ${ }^{1}$ \\ Xavier Charpentier, ${ }^{1}$ Mamadou Daffé ${ }^{1}$ and Annaïk Quémard ${ }^{1}$
}

\footnotetext{
1 Institut de Pharmacologie et de Biologie Structurale, Centre National de la Recherche Scientifique, Université Paul Sabatier (UMR5089), 205 route de Narbonne, 31077 Toulouse cedex, France

2 Centre de Biochimie Structurale (INSERM U554 - CNRS UMR5048 - UM1), 29 rue de Navacelles, 34090 Montpellier cedex, France
}

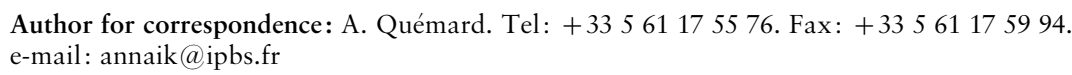

The fatty acid elongation system FAS-II is involved in the biosynthesis of mycolic acids, which are very long-chain fatty acids of the cell envelope specific to Mycobacterium tuberculosis and other mycobacteria. A potential component of FAS-II, the protein MabA (FabG1), was overexpressed and purified. Sedimentation equilibrium analyses revealed that MabA undergoes a dimer to tetramer self-association with a dissociation constant of $22 \mu \mathrm{M}$. The protein was detected by Western blotting in a mycobacterial cell-wall extract that produces mycolic acids and in the FPLC FAS-II fraction. MabA was shown to catalyse the NADPH-specific reduction of $\beta$-ketoacyl derivatives, equivalent to the second step of a FAS-II elongation round. Unlike the known homologous proteins, MabA preferentially metabolizes long-chain substrates $\left(C_{8}-C_{20}\right)$ and has a poor affinity for the $C_{4}$ substrate, in agreement with FAS-II specificities. Molecular modelling of MabA structure suggested the presence of an unusually hydrophobic substrate-binding pocket holding a unique Trp residue, suitable for fluorescence spectroscopic analyses. In agreement with the enzyme kinetic data, the spectral properties of MabA were different in the presence of the $C_{8}-C_{16}$ ligands as compared to the $C_{4}$ ligand. Altogether, these data bring out distinctive enzymic and structural properties of MabA, which correlate with its predilection for long-chain substrates, in contrast to most of the other known ketoacyl reductases.

Keywords: $\beta$-ketoacyl reductase, quaternary structure, enzymic activity, structural model, fluorescence spectroscopy

\section{INTRODUCTION}

Mycolic acids, very long-chain fatty acids $\left(\mathrm{C}_{60}-\mathrm{C}_{90}\right)$, play an essential role in the architecture and permeability of the envelope of mycobacteria, including Mycobacterium tuberculosis (Liu et al., 1996; Daffé \& Draper, 1998). The front-line antituberculous drug isoniazid

\footnotetext{
†Present address: St Jude Children's Research Hospital, Dept of Biochemistry, 322 N Lauderdale, Memphis, TN 38105, USA.

¥ Hedia Marrakchi and Stéphanie Ducasse contributed equally to this work. Abbreviations: ACP, acyl carrier protein; ESI, electrospray ionization; FAS, fatty acid synthetase; H-MabA, His-tagged MabA; INH, isoniazid MALDI-TOF, matrix-associated laser desorption ionization-time of flight; $\mathrm{KAR}, \beta$-ketoacyl-ACP reductase; PDB, Protein Data Bank; SDR, short-chain dehydrogenases/reductases.
}

(INH) impairs biosynthesis of these $\alpha$-branched and $\beta$-hydroxylated molecules by inhibiting the production, by the fatty acid elongation system called FAS-II, of medium- to long-chain fatty acids, which would be precursors of mycolic acids (Takayama et al., 1972; Quémard et al., 1991; Marrakchi et al., 2000; Vilchèze et al., 2000). Indeed, INH inhibits a 2-transenoyl-acyl carrier protein (ACP) reductase (ENR), called InhA (Quémard et al., 1996; Zabinski \& Blanchard, 1997; Rozwarski et al., 1998), that catalyses the last step of the four-step elongation rounds performed by FAS-II (Marrakchi et al., 2000). InhA has been shown to be an essential enzyme for mycobacterial viability (Vilchèze $e t$ al., 2000), leading to the conclusion that the production of long-chain fatty acids and mycolic acids is essential for mycobacterial growth. It has been suggested that the 
KasA protein, which catalyses the first step of FAS-II elongation rounds, would also be a target of INH (Mdluli et al., 1998). However, it has recently been shown that overexpression of the kasA gene did not modify the INH-susceptibility level of the vaccine strain $M$. bovis BCG, and mutations found in the kas $A$ gene of INH-resistant clinical isolates are also present in INHsusceptible strains (Kremer et al., 2000).

The FAS-II system has been isolated from a nonpathogenic mycobacterium, M. smegmatis (Odriozola et al., 1977). It is a complex of several monofunctional enzymes that catalyses the ACP-dependent elongation of palmitoyl-CoA $\left(\mathrm{C}_{16}\right)$ into $\mathrm{C}_{18}-\mathrm{C}_{30}$ saturated fatty acids, using malonyl-CoA as an elongation unit (Bloch, 1977). The mycobacterial FAS-II is an unusual type II system in that it elongates medium-chain-length substrates, while the other known bacterial systems perform de novo biosynthesis (Bloch, 1977). Only some of the proteins forming FAS-II have been positively identified, namely ACP (Bloch, 1977, Kremer et al., 2001), and more recently InhA, KasA and mtFabD (Marrakchi et al., 2000; Kremer et al., 2000, 2001). The inhA gene is assumed to form an operon together with the upstream ORF, mabA (fabG1) (Banerjee et al., 1994). The latter encodes a protein whose predicted amino acid sequence displays similarities with $\beta$-ketoacyl-ACP reductases (KARs or FabG), and a total soluble protein extract of Escherichia coli expressing mabA had KAR activity (Banerjee et al., 1998). Cloning, overexpression and purification were performed in order to obtain the pure MabA protein for both biochemical and biophysical characterizations. Here we report enzymological and structural analyses of MabA that represent a fundamental step towards the design of inhibitors. Specific structural features of MabA, interrelated with its substrate specificity, could allow the development of new antibiotics directed against mycobacteria.

\section{METHODS}

Cloning and overexpression of mabA. The mabA (fabG1, Rv1483) gene of $M$. tuberculosis H37Rv was amplified by PCR from the cosmid MTCY277 (provided by Dr. S. Cole, Institut Pasteur, Paris, France) using Pfu DNA polymerase (Stratagene) and primers which contained NdeI and XhoI restriction sites, respectively (5'-GTCCATATGACTGCCACAGCCACTG-3', forward primer, and 5'-GTCTCGAGCGTCCTTGTGTTGTGTC-3', reverse primer; restriction sites are underlined). PCR was performed for 10 cycles, in the presence of $5 \%(\mathrm{v} / \mathrm{v})$ formamide and $10 \%(\mathrm{v} / \mathrm{v})$ DMSO, at an annealing temperature of $60^{\circ} \mathrm{C}$. The PCR product was ligated with T4 DNA ligase (Promega) downstream of a His-Tag oligohistidine-encoding sequence into the pET-15b overexpression vector (Novagen), which had been previously treated with NdeI and XhoI restriction enzymes. This construct was used to transform E. coli BL21(DE3) (Novagen). The absence of mutation was verified by sequencing the cloned mabA gene. The recombinant strain was grown at $37{ }^{\circ} \mathrm{C}$ in a rotary shaker (250 r.p.m.) in $200 \mathrm{ml}$ Luria broth supplemented with $50 \mu \mathrm{g}$ carbenicillin $\mathrm{ml}^{-1}$. Expression of mabA was induced by the addition of $0.8 \mathrm{mM}$ IPTG at an $\mathrm{OD}_{600}$ of $0 \cdot 8-0 \cdot 9$, and a further $2 \mathrm{~h}$ incubation at $37^{\circ} \mathrm{C}$.
Purification of MabA protein. Induced recombinant bacteria were washed with cold $50 \mathrm{mM}$ potassium phosphate buffer $\mathrm{pH} 7 \cdot 8$ (buffer A), and resuspended in $4 \mathrm{ml}$ of the same buffer supplemented with $500 \mathrm{mM} \mathrm{NaCl}$ and $5 \mathrm{mM}$ imidazole. Cells were broken by one freezing/thawing cycle at $-70{ }^{\circ} \mathrm{C}$, in the presence of protease inhibitors [aprotinin, soybean trypsininhibitor, TLCK (N-p-tosyl-lysine chloromethyl ketone), pepstatin, leupeptin and PMSF] and $0.5 \mathrm{mg}$ lysozyme $\mathrm{ml}^{-1}$. Nucleic acids were removed by DNase I $\left(5 \mu \mathrm{g} \mathrm{ml}^{-1}\right)$ and RNase A $\left(10 \mu \mathrm{g} \mathrm{ml}^{-1}\right)$ treatments in the presence of $\mathrm{MgCl}_{2}(10$ $\mathrm{mM})$ at $4{ }^{\circ} \mathrm{C}$ for $15 \mathrm{~min}$. After centrifugation at $44000 \mathrm{~g}$ for $15 \mathrm{~min}$, the supernatant supplemented with $10 \%$ (v/v) glycerol was applied to a Ni-NTA Agarose (Qiagen) column $(0.5 \mathrm{ml}$ bed volume). After extensive washes by $5 \mathrm{mM}$ then $50 \mathrm{mM}$ imidazole in buffer A supplemented with $500 \mathrm{mM}$ $\mathrm{NaCl}, \mathrm{MabA}$ was eluted with $175 \mathrm{mM}$ imidazole in buffer A supplemented with $500 \mathrm{mM} \mathrm{NaCl}$. Fractions containing the His-tagged MabA (H-MabA) were identified by SDS-PAGE, pooled, dialysed twice against 50 vols $50 \%(\mathrm{v} / \mathrm{v})$ glycerol in $50 \mathrm{mM}$ potassium phosphate buffer, $\mathrm{pH} 7 \cdot 2$, and stored at $-20{ }^{\circ} \mathrm{C}$.

The InhA protein from M. tuberculosis was overproduced in E. coli and purified as previously described (Quémard et al., 1995).

Mass spectrometry (MS). Electrospray ionization (ESI) analysis of MabA was performed using a TSQ 700 (Finnigan MAT) quadrupole mass spectrometer. The protein was dissolved in methanol/water/acetic acid $(50: 49 \cdot 5: 0 \cdot 5$, by vol.) and introduced by a syringe pump (Harvard) at a flow rate of $5 \mu \mathrm{min}^{-1}$ into the electrospray source $\left(5 \mathrm{kV}, 250^{\circ} \mathrm{C}\right)$. Nitrogen (pressure 40 p.s.i., $276 \mathrm{kPa}$ ) was used as nebulizing gas. MALDI-TOF spectra (in the positive mode) were acquired on a Voyager-DE STR Biospectrometry workstation (PerSeptive Biosystems), equipped with a pulsed nitrogen laser emitting at $337 \mathrm{~nm}$, as described by Laval et al. (2001), using an extraction voltage of $20 \mathrm{kV}$ and 2,5-dihydroxybenzoic acid solution $\left[10 \mathrm{mg} \mathrm{ml}^{-1}\right.$ in water/acetonitrile, $8: 2(\mathrm{v} / \mathrm{v})]$ as matrix.

Analytical size-exclusion chromatography. A prepacked Sephacryl S-100HR column (Amersham Pharmacia Biotech), monitored by a BioCAD SPRINT system (PerSeptive Biosystems), was equilibrated in $50 \mathrm{mM}$ potassium phosphate buffer, pH 6.8, containing $100 \mathrm{mM} \mathrm{NaCl}$. H-MabA (0.7 mg) and InhA $(1.1 \mathrm{mg})$ were separately eluted through the column with the same buffer, at a flow rate of $0.5 \mathrm{ml} \mathrm{min}{ }^{-1}$. The column was calibrated in the same elution conditions by applying, in two separate elutions, the following standard proteins $(0 \cdot 5-1 \mathrm{mg}$ of each protein): alcohol dehydrogenase $(150 \mathrm{kDa})$, bovine serum albumin $(67 \mathrm{kDa})$, ovalbumin $(43 \mathrm{kDa})$, carbonic anhydrase $(29 \mathrm{kDa})$ and RNase A $(13.7 \mathrm{kDa})$. The molecular mass of $\mathrm{H}-\mathrm{MabA}$ was estimated from the calibration curve of elution volumes versus molecular masses.

Analytical ultracentrifugation. Analytical ultracentrifugation experiments were performed in a Beckman Optima XL-I analytical ultracentrifuge using an An-55 four-hole rotor with six-channel Epon charcoal-filled centrepieces, and absorbance optics. Prior to the measurements, the protein samples were eluted through a Fast desalting column HR 10/10 (Amersham Pharmacia Biotech) in $20 \mathrm{mM}$ MES, pH 6.4, $300 \mathrm{mM} \mathrm{LiSO}_{4}$ and $1 \mathrm{mM}$ tris-(carboxyethyl)phosphine hydrochloride. Sedimentation equilibrium experiments were carried out at $4{ }^{\circ} \mathrm{C}$ on sample volumes of $110 \mu \mathrm{l}$, at loading MabA concentrations of 26,39 and $78 \mu \mathrm{M}$, and at rotor speeds of 4872,7015 and $14637 \boldsymbol{g}$ (respectively 7500, 9000 and 13000 r.p.m.). Data were 
collected at $280 \mathrm{~nm}$ and runs were continued until there was no significant difference in scans taken $2 \mathrm{~h}$ apart. Data were analysed with the XL-A/XL-I Data Analysis software version 4.0 (Beckman). The partial specific volume $\left(0.7223 \mathrm{ml} \mathrm{g}^{-1}\right)$ of the recombinant $\mathrm{H}-\mathrm{MabA}$ protein and the solvent density $\left(1.04 \mathrm{~g} \mathrm{ml}^{-1}\right)$, at the experimental temperature, were calculated with help of the program Sednterp (Sedimentation Interpretation Program, version 1.05).

Preparation of FAS-I and FAS-II systems or cell-wall extract, and Western blots. Cell-wall extract, FAS-I and FAS-II systems were prepared from M. smegmatis $\mathrm{mc}^{2} 155$ as previously described (Marrakchi et al., 2000). An aliquot of total protein extract was kept after sonication of bacteria, during preparation of FAS-I and FAS-II systems. Samples were separated by $12 \%$ or $15 \%$ (w/v) polyacrylamide SDS-PAGE, and proteins transferred onto a nitrocellulose membrane. Polyclonal rabbit antibodies against pure $\mathrm{H}-\mathrm{MabA}$ protein were used for Western blot analyses. Rabbit preimmune serum was used for control experiments. Antigen-antibody interactions were revealed by colorimetric reaction, using alkaline phosphatase-mouse anti-rabbit IgG conjugates.

Synthesis of different chain length $\beta$-ketoacyl-CoAs. $\beta$ Ketoacyl-CoAs possessing 8-20 carbon atoms were synthesized as described by Vagelos \& Alberts (1960), with some modifications. For solubility reasons, for the $C_{16}$ and $C_{20}$ derivatives the deprotection step of the ketone function was realized by a transcetalization reaction, by incubating the ethylenic ketal derivatives in anhydrous acetone and p-toluenesulphonic acid $(10 \mathrm{mM})$ at $56^{\circ} \mathrm{C}$, for $36 \mathrm{~h}$. The derivatization into acyl-CoAs was performed at $\mathrm{pH} 8.7$, in $600 \mathrm{mM}$ Tris/tetrahydrofuran $(1: 1, \mathrm{v} / \mathrm{v})$. The compounds obtained at each synthesis step were purified and their structure characterized by electron-impact MS, IR and NMR spectroscopies. The final $\beta$-ketoacyl-CoA products were purified by chromatography on a $\mathrm{C}_{18}$ Sep-Pak cartridge equilibrated with $20 \mathrm{mM} \mathrm{NaH} \mathrm{PO}_{4}$ and elution with a $0-80 \%$ methanol gradient in water, and their structures verified by ESI-MS in the negative mode.

Enzyme assays and steady-state kinetics. Kinetic parameters were determined spectrophotometrically by following NADPH oxidation at $340 \mathrm{~nm}$ using a thermostatted Uvikon 923 spectrophotometer (Kontron Instruments). Standard reactions were performed in a quartz cuvette in a total volume of $1 \mathrm{ml}$, at $25^{\circ} \mathrm{C}$, in $100 \mathrm{mM}$ sodium phosphate buffer, $\mathrm{pH} 7 \cdot 0$, in the presence of fixed concentrations of NADPH and $\beta$ ketoacyl-CoA; after equilibration of the baseline, reactions were started by adding a defined amount of H-MabA enzyme, and measurements performed for 3-5 min. Comparison of the initial reaction rates obtained for the different chain length $\beta$ ketoacyl-CoAs at a low substrate concentration was performed at $2 \mu \mathrm{M} \beta$-ketoacyl-CoA, $100 \mu \mathrm{M}$ NADPH and 18-360 nM H-MabA (depending on the substrate used). The steadystate $K_{\mathrm{m}}$ value for NADPH was determined by measuring initial velocities at various concentrations of coenzyme and at fixed concentrations of acetoacetyl-CoA $(400 \mu \mathrm{M})$ and of enzyme $(36 \mathrm{nM})$. The $K_{\mathrm{m}}$ values for $\beta$-ketoacyl-CoAs and $k_{\text {cat }}$ values were measured by varying the concentration of each $\beta$ ketoacyl-CoA and at fixed concentrations of NADPH $(100 \mu \mathrm{M})$ and of enzyme $(18-144 \mathrm{nM}$, depending on the substrate). Data were fitted to the Michaelis-Menten equation by least-squares fits to a hyperbola using the program GraphPad Prism to calculate the kinetic parameters. For MALDI-TOF MS analyses, reactions were performed in a total volume of $1 \mathrm{ml}$, at $25^{\circ} \mathrm{C}$, in the presence of $300 \mu \mathrm{M}$ NADPH, $200 \mu \mathrm{M}$ acetoacetyl-CoA and $800 \mathrm{nM} \mathrm{H}-\mathrm{MabA}$, in
$20 \mathrm{mM}$ Tris buffer, $\mathrm{pH} 7 \cdot 0$, and stopped after completion (monitored by $A_{340}$ measurement).

Sequence comparison and molecular modelling. Protein sequence database searches were performed with PSI-BLAST version 2.0.5 (Altschul et al., 1997), with default parameters. Alignment refinement was subsequently performed using the program TITO (Labesse \& Mornon, 1998) and structures of related enzymes (see text). MabA secondary structures $(\alpha$ helices, $\beta$-strands) were assigned during TITo processing, and the secondary structures derived by homology were used as additional restraints in the following modelling step (except for the stretch 48-GSGAPKG-54 due to its sequence enriched in glycine). The three-dimensional model was built using the quaternary structure deduced from the crystal structure of PDB1EDO $(40 \%$ identical) as a template in the program MODELLER 4.0 (Sali \& Blundell, 1993) and assessed using Verify-3D (Eisenberg et al., 1997) and prosa (Sippl, 1993). These three-dimensional structures were visualized on a UNIX workstation using the program InsightII (MSI, San Diego, USA).

Fluorescence spectroscopy. Steady-state emission spectra were recorded on an ISS K2 spectrofluorimeter through a $8 \mathrm{~nm}$ bandwidth monochromator and corrected for buffer emission. Time-resolved data were collected in the frequency domain using ISS acquisition electronics and analysed with the software Globals Unlimited (Beechem et al., 1991). The excitation light was at $300 \mathrm{~nm}$ from the frequency-tripled output of a pulse-picked Tsunami Ti-Sa laser pumped by a Millennia X dye laser (Spectra Physics). The reference lifetime compound was $\mathrm{N}$-acetyltryptophanamide in water (3 ns). Measurements were carried out using $6 \mu \mathrm{M} \mathrm{H}-\mathrm{MabA}$ in $100 \mathrm{mM}$ sodium phosphate buffer, $\mathrm{pH} 7 \cdot 0$, and $7 \%(\mathrm{w} / \mathrm{v})$ glycerol, in the presence or absence of a ligand (at saturating concentration of $\mathrm{NADP}^{+}$and/or $K_{\mathrm{m}}$ concentration of $\beta$ ketoacyl-CoA). Fluorescence spectroscopy in the presence of $\mathrm{NADPH}$ was not performed as the coenzyme absorption spectrum overlaps the emission spectrum of the protein tryptophan residue.

\section{RESULTS}

\section{Expression of the $M$. tuberculosis MabA protein in $E$. coli and purification}

The mabA gene of M. tuberculosis was cloned into the plasmid pET-15b. The N-terminal His-tagged MabA protein (H-MabA) was produced at high levels $(30 \%$ of the total soluble proteins) in E. coli BL21(DE3), and purified to greater than $96 \%$ purity by chromatography over a Ni-NTA agarose column (Fig. 1). After dialysis, the amount of purified protein recovered corresponded to the quasi-total amount of H-MabA loaded onto the column. ESI-MS analysis showed the mass of the protein to be $27729 \mathrm{Da}$, which is $131 \mathrm{Da}$ below the predicted average mass for H-MabA (27860: 25697+2163). Amino acid sequencing revealed that this mass difference corresponds to the loss of the methionine at the $\mathrm{N}$ terminus of the recombinant protein.

\section{Quaternary structure of MabA}

The quaternary structure of MabA was analysed by gel filtration. The chromatogram suggested the presence of two molecular species (a major peak at $95 \mathrm{kDa}$ and a 


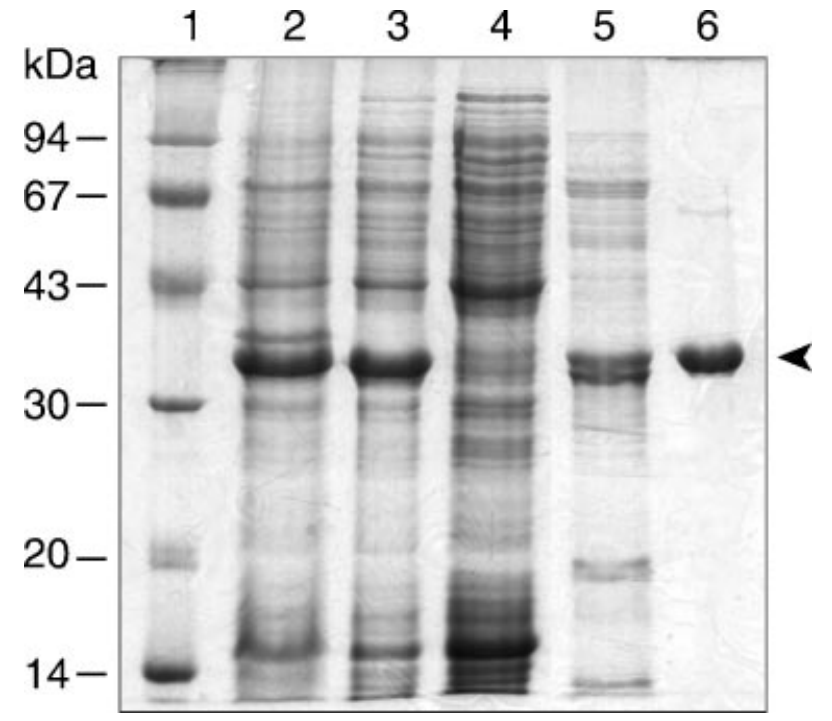

Fig. 1. Expression of $\mathrm{H}-\mathrm{MabA}$ protein in $E$. coli and purification. SDS-PAGE analysis of $25 \mu \mathrm{g}$ of each sample is shown: (1) molecular mass markers, (2) crude extract of E. coli BL21(DE3)/ $h$-mabA treated with IPTG, (3) total soluble protein extract of $E$. coli BL21(DE3)/h-mabA loaded onto the Ni-NTA agarose column, (4) first wash with $5 \mathrm{mM}$ imidazole, (5) first wash with $50 \mathrm{mM}$ imidazole, (6) pooled fractions after elution of $\mathrm{H}$-MabA with $175 \mathrm{mM}$ imidazole. The gel was stained with Coomassie blue.

minor one at $58 \mathrm{kDa})$. The InhA protein $(114 \mathrm{kDa})$, used as a reference, eluted as a single symmetrical peak estimated at $111 \mathrm{kDa}$, in agreement with a previous report (Rozwarski et al., 1999). Sedimentation equilibrium analyses displayed an increase of the apparent weight-average molecular weight $\left(M_{\mathrm{w}, \mathrm{app}}\right)$ with absorbance, indicating the behaviour of a self-associating system of several molecular species (data not shown). The maximum value of $M_{\mathrm{w} \text {,app }}$ (around $100 \mathrm{kDa}$ ) suggested that the largest species was a tetramer $(110 \cdot 9 \mathrm{kDa})$. When datasets were analysed separately by non-linear least-squares fittings, best fits were obtained for the dimer-tetramer model (Fig. 2). The other models gave variances and $95 \%$ confidence intervals that were too large, systematic errors on the residuals plots or aberrant $K_{\mathrm{d}}$ values. Global simultaneous fittings of all datasets gave a dimer-tetramer equilibrium association constant of $7.9 \pm 0.7 \mathrm{ml} \mathrm{mg}^{-1}$, corresponding to a $K_{\mathrm{d}}$ of $22 \pm 2 \mu \mathrm{M}$. Thus, gel filtration chromatography and sedimentation equilibrium experiments both showed that MabA can self-associate in a tetrameric structure, as observed for KARs from plants (Sheldon et al., 1990, 1992; Fisher et al., 2000).

\section{Coelution of MabA with the FAS-II system}

MabA was detected by Western blotting in a mycobacterial cell-wall extract (Fig. 3b) that produces mycolic acids and contains the FAS-II complex (Marrakchi et al., 2000). The extra band observed may be linked to proteolysis of MabA or to cross-reactivity of the

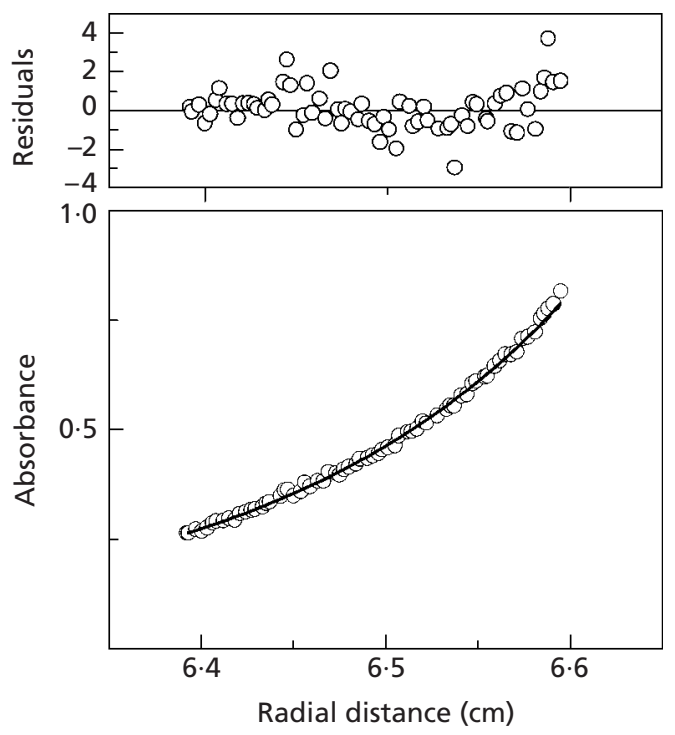

Fig. 2. Equilibrium sedimentation of MabA. The upper part of the panel shows the residual difference between experimental and fitted values by its standard deviation. This experiment was carried out at three different centrifugation speeds and three different protein concentrations (see Methods), and was repeated twice using distinct protein batches. An identical fit was performed for all datasets, but only the result obtained with $78 \mu \mathrm{M}$ MabA at 9000 r.p.m. is shown here for more clarity.
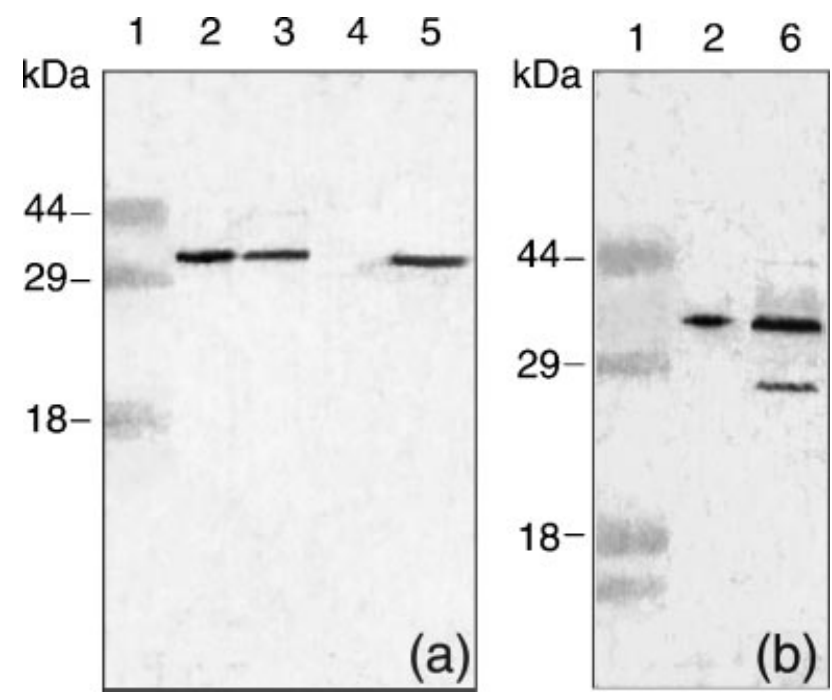

Fig. 3. Detection of MabA in M. smegmatis protein fractions by Western blotting. (a) Analysis of FAS-I and FAS-II. (b) Analysis of cell-wall extract. Lanes: (1) pre-stained molecular standards, (2) pure $\mathrm{H}-\mathrm{MabA}$, (3) total sonication extract from $M$. smegmatis, (4) fraction FAS-I, (5) fraction FAS-II, (6) cell-wall extract. For SDS-PAGE, $25 \mu \mathrm{g}$ of proteins of each sample and $10 \mathrm{ng}$ of pure $\mathrm{H}-\mathrm{MabA}$ were used. For Western blotting, polyclonal rabbit anti-MabA antibodies were used. No signal was obtained using the rabbit preimmune serum (data not shown).

antibodies with another KAR, since there are four other putative KARs in M. tuberculosis. The mycobacterial fatty acid biosynthesis systems FAS-I and FAS-II were 


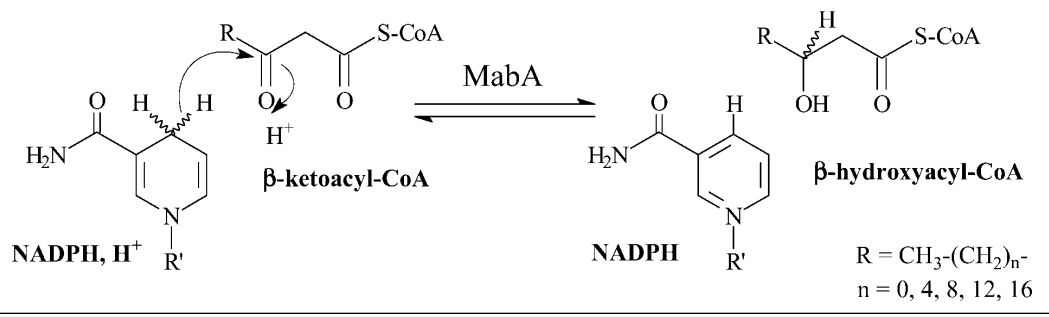

Fig. 4. Reaction scheme for the reduction of $\beta$-ketoacyl-CoA substrates by MabA.

Table 1. Kinetic parameters of the MabA protein

Reactions were run at $25^{\circ} \mathrm{C}$, in $100 \mathrm{mM}$ sodium phosphate buffer, $\mathrm{pH} 7 \cdot 0$, and were followed spectrophotometrically at $340 \mathrm{~nm}$ (see Methods). Data were fitted by non-linear least-squares regression to calculate the kinetic parameters. Values are means of three separate experiments \pm SD.

\begin{tabular}{|lccc|}
\hline Substrate & $\boldsymbol{K}_{\mathrm{m}}(\boldsymbol{\mu} \mathrm{M})$ & $\boldsymbol{k}_{\mathrm{cat}}\left(\mathbf{s}^{-\mathbf{1}}\right)$ & $\boldsymbol{k}_{\mathrm{cat}} / \boldsymbol{K}_{\mathrm{m}}\left(\mathbf{s}^{-\mathbf{1}} \boldsymbol{\mu \mathbf { M } ^ { - 1 } )}\right.$ \\
\hline NADPH & $41 \pm 7$ & $2 \cdot 6 \pm 0 \cdot 2$ & $0 \cdot 063 \pm 0 \cdot 015$ \\
Acetoacetyl-CoA $\left(\mathrm{C}_{4}\right)$ & $1530 \pm 81$ & $1 \cdot 9 \pm 0 \cdot 0$ & $0 \cdot 001 \pm 0 \cdot 000$ \\
$\beta$-Ketooctanoyl-CoA $\left(\mathrm{C}_{8}\right)$ & $70 \pm 8$ & $3 \cdot 5 \pm 0 \cdot 2$ & $0 \cdot 050 \pm 0 \cdot 008$ \\
$\beta$-Ketododecanoyl-CoA $\left(\mathrm{C}_{12}\right)$ & $8 \cdot 3 \pm 0 \cdot 8$ & $4 \cdot 3 \pm 0 \cdot 2$ & $0 \cdot 520 \pm 0 \cdot 074$ \\
\hline
\end{tabular}

isolated by FPLC, and Fig. 3(a) shows that MabA coeluted with FAS-II, but not with FAS-I.

\section{Enzymic activity of the MabA protein}

The reduction of $\beta$-ketoacyl derivatives by the recombinant purified protein (Fig. 4) was monitored by spectrophotometry. It showed that $\mathrm{H}-\mathrm{MabA}$ is active in the simultaneous presence of acetoacetyl-CoA $\left(\mathrm{C}_{4}\right.$ substrate) and NADPH. MALDI-TOF MS analyses showed that the protein reduces acetoacetyl-CoA $\left([\mathrm{M}+\mathrm{H}]^{+}\right.$at $m / z 852,[M+\mathrm{Na}]^{+}$at $\left.m / z 874\right)$ to hydroxybutyryl$\mathrm{CoA}\left([M+\mathrm{H}]^{+}\right.$at $m / z 854,[M+\mathrm{Na}]^{+}$at $\left.m / z 876\right)$. This activity was linearly dependent on the concentration of $\mathrm{H}-\mathrm{MabA}$, and was not detected when NADPH was replaced by $\mathrm{NADH}$, indicating that the enzyme is NADPH-dependent, like most of the known KARs (Bloch, 1977). In the absence of NADPH, the production of saturated fatty acids by the FAS-II system was totally abolished, while $\beta$-ketoesters accumulated (data not shown), consistent with MabA being a key component of FAS-II.

Steady-state kinetic experiments (Table 1) gave a $K_{\mathrm{m}}$ value of MabA for acetoacetyl-CoA of $1530 \mu \mathrm{M}$, which is 6 times the $K_{\mathrm{m}}$ of KARs from plants (Sheldon et al., 1992; Shimakata \& Stumpf, 1982). In contrast, the $k_{\text {cat }}$ and the $K_{\mathrm{m}}$ for NADPH reported for these proteins are of the same order of magnitude as those measured for MabA (Table 1).

To determine the substrate specificity of the protein, longer-chain $\beta$-ketoacyl-CoAs were synthesized. The measured kinetic parameters revealed a much better activity of MabA with long-chain substrates, since the $K_{\mathrm{m}}$ and the $k_{\text {cat }}$ significantly improved with $\mathrm{C}_{8}$ and $\mathrm{C}_{12}$ derivatives (Table 1). The kinetic parameters for the $\mathrm{C}_{16}$ and $\mathrm{C}_{20}$ substrates could not be determined because of an inhibition phenomenon at high substrate concentration, which has been observed for other fatty acid biosynthesis systems (Bloch, 1977). Initial reaction rates were nevertheless compared at a very low substrate concentration $(2 \mu \mathrm{M})$, in the presence of $\beta$-ketoacylCoAs ranging from $C_{8}$ to $C_{20}$. The highest values of initial velocity were obtained with the $\mathrm{C}_{12}$ and $\mathrm{C}_{16}$ substrates $\left[V_{\mathrm{i}}\right.$ values of $0 \cdot 7 \pm 0 \cdot 1$ and $0 \cdot 4 \pm 0 \mu \mathrm{mol} \mathrm{min}{ }^{-1}$ $(\mathrm{mg} \text { protein })^{-1}$, respectively]; moreover, the $\mathrm{C}_{20}$ derivative appeared to be a better substrate than the $\mathrm{C}_{8}$ one (respectively 23 and $13 \%$ of the $V_{i}$ for $C_{12}$ ). These data showed that MabA has a predilection for long-chain substrates.

\section{Structural analysis of the MabA protein}

Through PSI-BLAST searches (Altschul et al., 1997) performed in non-redundant databases, MabA appeared highly conserved among mycobacteria $(81-84 \%$ identity in M. tuberculosis, M. avium and M. smegmatis), while identity scores with other KARs (16 sequences) from various organisms, bacteria or plants, ranged from 29 to $43 \%$ over the whole sequence. The search for related three-dimensional structures indicated extended similarities with the short-chain dehydrogenases/reductases (SDR) superfamily (Rafferty et al., 1995; Jörnvall et al., 1995), or the reductases/epimerases/dehydrogenases (RED) superfamily (Labesse et al., 1994), to which InhA belongs, the closest structure being the KAR from Brassica napus (PDB1EDO), with $40 \%$ identity. Most residues critical for cofactor binding and catalysis are well conserved in MabA sequence (Fig. 5). Furthermore, in agreement with the kinetic data, the $\beta 2$ strand displays 


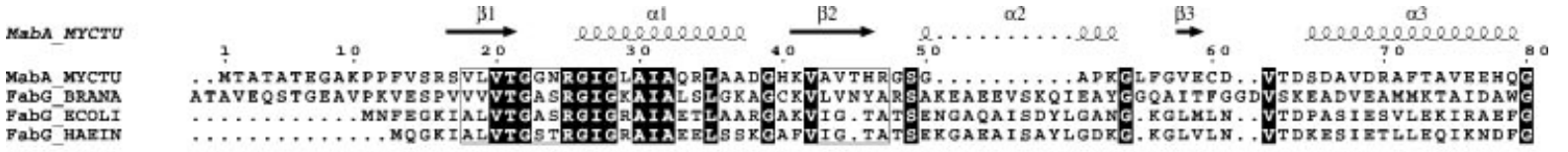

Barm

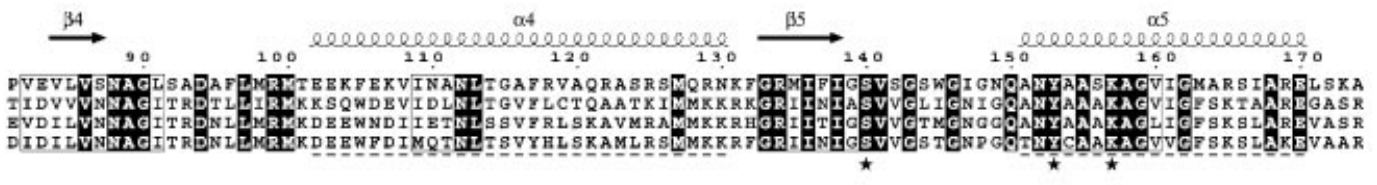

Mabs MYCTU

Maba_ ryctu

FabG BCOLI

FabG HAEIN

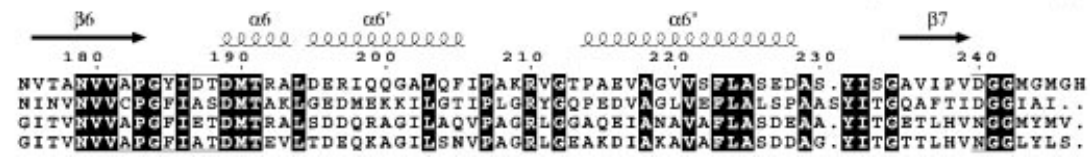

Fig. 5. Alignment of the MabA sequence with that of KARs from B. napus, E. coli and Haemophilus influenzae. The alignment was performed with CLUSTAL W (Thompson et al., 1994). Strictly conserved residues are highlighted with black boxes. Secondary-structure elements corresponding to MabA protein are drawn on the top of the alignment. The consensus sequences defining the SDR superfamily signature (Jörnvall et al., 1995; Labesse et al., 1994) are framed. Stars indicate the amino acids forming the catalytic triad; dotted lines indicate conserved motifs involved in the tetramer interfaces. The figure was drawn with the program ESPript (Gouet et al., 1999).
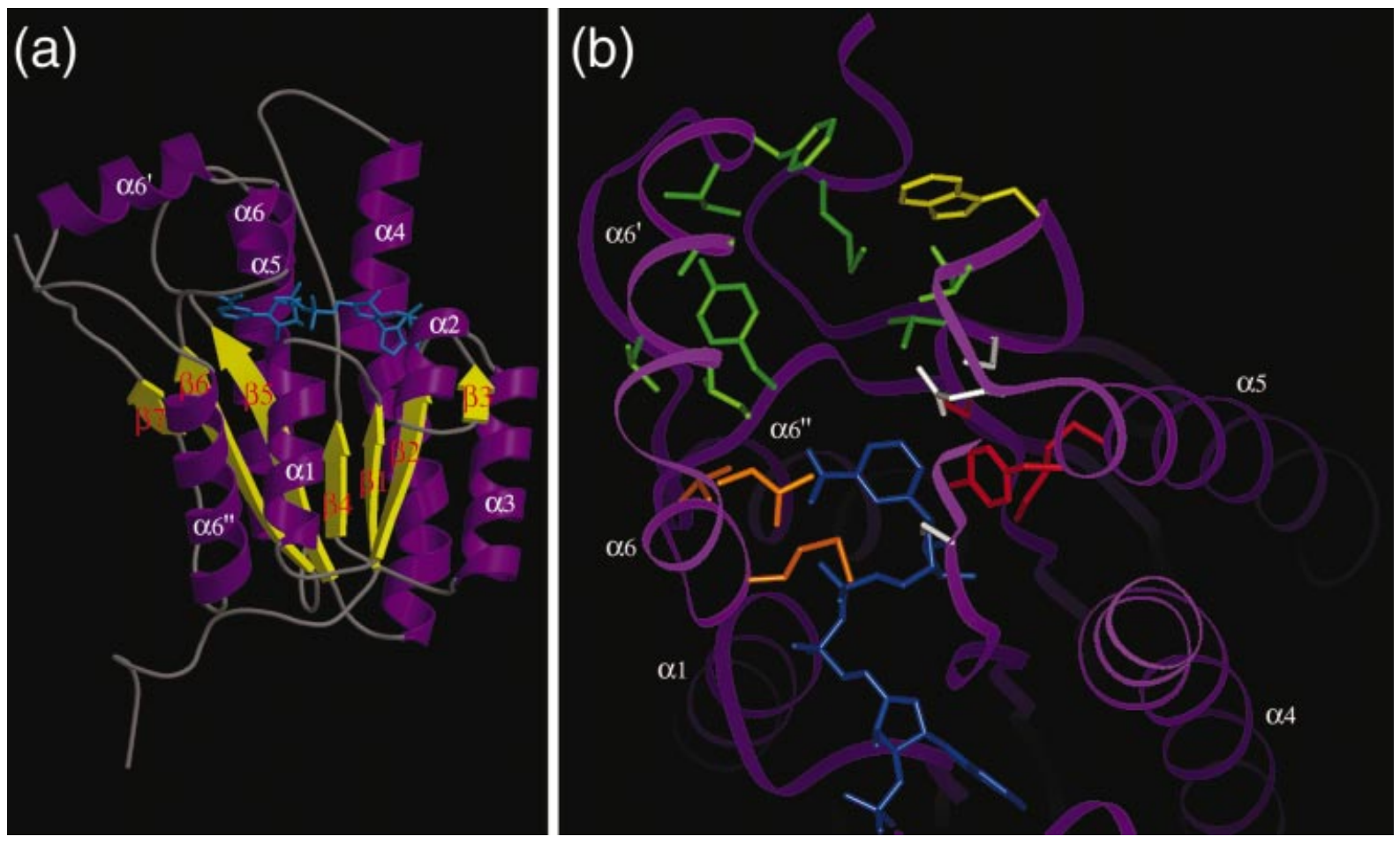

Fig. 6. Structural model of a MabA subunit complexed with one NADPH molecule. The trace of the amino acid chain is displayed as a ribbon. Secondary-structure elements are labelled. NADPH is drawn as blue sticks. (a) Complete view of the subunit. $\alpha$-Helices are coloured in purple and $\beta$-strands in yellow. The cavity delimited by the C-termini of the $\beta 4, \beta 5, \beta 6$, $\beta 7$ strands and the $\alpha 4, \alpha 5, \alpha 6, \alpha 6^{\prime}$ and $\alpha 6^{\prime \prime}$ helices correspond to the predicted substrate-binding site. (b) Top view of the predicted active site of MabA. A rotation of 90 degrees compared to the view in (a) was performed. The trace of the amino acid chain is coloured in purple. The side chains of selected amino acid residues are shown. Hydrophobic residues at the bottom of the substrate-binding pocket are coloured in green (Val ${ }^{141}, \| e^{147}, \mathrm{Tyr}^{185}, \mathrm{Il}^{198}, \mathrm{Gly}^{201}, \mathrm{Ala}^{202}, \mathrm{Phe}^{205}$, $\| \mathrm{e}^{206}, \mathrm{Val}^{211}$, Met ${ }^{243}$ ), except for $\operatorname{Trp}^{145}$, which is in yellow. The catalytic triad is coloured in red (Ser ${ }^{140}, \operatorname{Tyr}^{153}$, Lys $^{157}$ ), polar residues surrounding the triad are in white $\left(\operatorname{Ser}^{92}, \operatorname{Ser}^{142}, \mathrm{Gln}^{150}\right)$, and hydrophobic residues facing the triad are in orange (Met ${ }^{190}$, Thr ${ }^{191}$, Leu ${ }^{194}$ ). Panel (a) was produced using the MolScript program (Kraulis, 1991), and panel (b) using the Insightll program (MSI). 


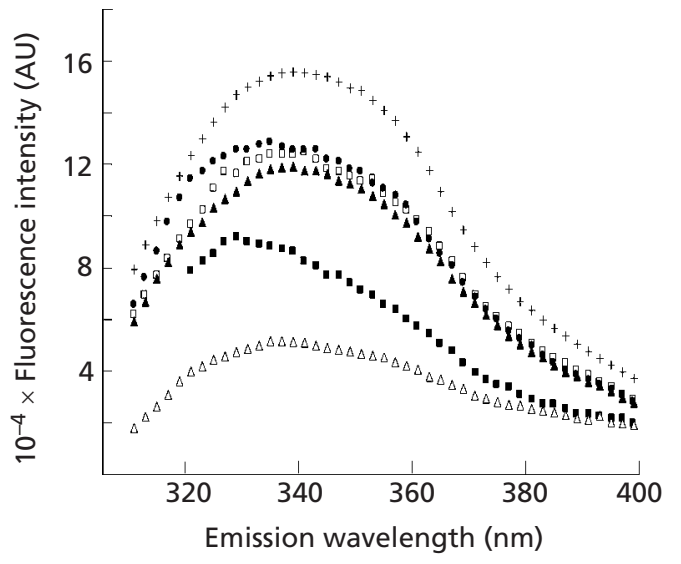

Fig. 7. Fluorescence emission of MabA in the presence of different ligands. Emission spectra in the absence of ligand $(+)$ or in the presence of $\beta$-ketoacyl-CoAs $C_{4}(\triangle), C_{8}(\Delta), C_{12}(\mathbf{O})$, $C_{16}(\square)$ or $\operatorname{NADP}^{+}(\square)$. Excitation wavelength: $300 \mathrm{~nm}$. AU, arbitrary unit. Similar data were obtained for the substrates in the presence of NADP'.

a sequence motif (Fig. 5) typical of the $\operatorname{NADP}(\mathrm{H})$ specific enzymes (Labesse et al., 1994).

MabA protein structure was modelled with good scores [PROSA (Sippl, 1993), - 1.50; Verify-3D (Eisenberg et al., 1997), $+0 \cdot 45$; versus $-1 \cdot 65$ and $+0 \cdot 55$, respectively, for the template] using the structure of PDB1EDO in complex with NADPH (Fisher et al., 2000). The two mostly hydrophobic interfaces of dimerization (Fig. 5) found in the tetrameric SDR proteins (Rafferty et al., 1995; Jörnvall et al., 1995; Fisher et al., 2000), are conserved in the MabA model. The nicotinamide portion of NADPH, involved in hydride transfer during catalysis (Fig. 4), points deep into the substrate-binding site of the protein (Fig. 6a). Three distinct parts may be described in this pocket (Fig. 6b). A patch of polar residues that surrounds the catalytic triad, and a mainly hydrophobic region facing the catalytic triad (Fig. 6b) are very well conserved among the known KARs (except
Ser ${ }^{142}$, which is often replaced by a residue of the same size, a Val) (Fig. 5). The bottom of the MabA substratebinding pocket appeared mainly hydrophobic, and the unique tryptophan residue $\left(\operatorname{Trp}{ }^{145}\right)$ was modelled in the vicinity of this cavity (Fig. 6b). Interestingly, in most other KARs, the hydrophobic residues $\operatorname{Trp}^{145}$ Ile $^{147}$, Ile $^{198}$ and Phe $^{205}$ observed in the MabA pocket are replaced by more polar residues (see Fig. 5). In contrast, these hydrophobic residues are perfectly conserved in MabA from M. avium and M. smegmatis. Furthermore, the very C-terminus $\left[{ }^{243} \mathrm{MGMGH}^{247}\right]$ of the mycobacterial MabA proteins is highly specific to this subclass of KARs (data not shown). These specific structural features might correlate with the observed distinct substrate specificity. Comparison of our MabA model with the crystal structure of the ternary complex InhA-NAD ${ }^{+}-\mathrm{C}_{16}$ substrate (Rozwarski et al., 1999) and manual fitting of the $\mathrm{C}_{16}$ substrate suggested that the MabA active site would accommodate the $\beta$-ketoacylCoA with minor rearrangements.

\section{Fluorescence spectroscopy}

The presence of a unique Trp residue in the MabA predicted substrate-binding pocket prompted us to test the effect of ligand binding on MabA fluorescence emission. Upon excitation at $300 \mathrm{~nm}$, the emission spectrum displayed a maximum at about $340 \mathrm{~nm}$ (Fig. 7), which showed that the tryptophan is partially buried (Lakowicz, 1983). Upon addition of $\mathrm{NADP}^{+}$, a diminution of fluorescence intensity and a blue-shifting of the $\lambda_{\max }$ (from 340 to $332 \mathrm{~nm}$ ) were observed (Fig. 7). This fluorescence quenching arose from a decrease of the populations of the long- and middle-lifetime emitting species (Table 2), despite a slight increase of the lifetime values, showing a conformational restraint of the Trp residue dynamics. These phenomena, together with an increase of the fluorescence polarization (data not shown), showed that the substrate-binding pocket closes upon NADP binding. In the presence of acetoacetylCoA, a similar behaviour was observed by steady-state spectroscopy (Fig. 7) and differential anisotropy (data

\section{Table 2. Time-resolved fluorescence of MabA}

Analysis of the frequency response profiles of the MabA tryptophan fluorescence in the absence or presence of different ligands, $\beta$ ketoacyl-CoAs or $\mathrm{NADP}^{+}$. Data were fitted using a triple exponential model and expressed as $\tau$ (fluorescence lifetime) values and as a percentage of emitting species for each lifetime (long, middle and short). These three lifetimes are expected for the three rotamers around the $\chi_{1}$ angle of a Trp residue within a protein. In each case, the chi-square for the fit is less than $0 \cdot 15$. Measurements were repeated in two independent experiments, and representative results are shown.

\begin{tabular}{|c|c|c|c|c|c|c|c|c|c|c|c|c|}
\hline \multirow{2}{*}{$\begin{array}{l}\text { Fluorescence } \\
\text { lifetime }\end{array}$} & \multicolumn{2}{|c|}{ No ligand } & \multicolumn{2}{|c|}{$\mathrm{C}_{4}$} & \multicolumn{2}{|c|}{$\mathrm{C}_{8}$} & \multicolumn{2}{|c|}{$\mathrm{C}_{12}$} & \multicolumn{2}{|c|}{$\mathrm{C}_{16}$} & \multicolumn{2}{|c|}{$\mathrm{NADP}^{+}$} \\
\hline & $\tau(\mathrm{ns})$ & $\%$ & $\tau(\mathrm{ns})$ & $\%$ & $\tau(\mathbf{n s})$ & $\%$ & $\tau(\mathbf{n s})$ & $\%$ & $\tau(\mathbf{n s})$ & $\%$ & $\tau(\mathbf{n s})$ & $\%$ \\
\hline Long & $7 \cdot 7$ & 14 & $5 \cdot 8$ & 8 & $7 \cdot 1$ & 18 & $7 \cdot 1$ & 18 & $7 \cdot 0$ & 19 & $9 \cdot 8$ & 4 \\
\hline Middle & $2 \cdot 8$ & 36 & $2 \cdot 1$ & 29 & $2 \cdot 7$ & 37 & $2 \cdot 7$ & 34 & $2 \cdot 2$ & 39 & $3 \cdot 5$ & 24 \\
\hline Short & $0 \cdot 5$ & 50 & $0 \cdot 3$ & 63 & $0 \cdot 5$ & 45 & $0 \cdot 5$ & 47 & $0 \cdot 4$ & 41 & $0 \cdot 5$ & 72 \\
\hline
\end{tabular}


not shown). In this case, however, the quenching arose from a decrease of all the three fluorescence lifetimes (Table 2), suggesting that the induced conformational change brings a quencher into the vicinity of the Trp residue.

Interestingly, replacement of acetoacetyl-CoA $\left(\mathrm{C}_{4}\right)$ by longer-chain substrates $\left(\mathrm{C}_{8}-\mathrm{C}_{16}\right)$, either in the presence or in the absence of $\mathrm{NADP}^{+}$, induced a significant release of quenching (Fig. 7). The fractional populations and fluorescence lifetimes of MabA alone were mostly recovered (Table 2), suggesting that the Trp residue and the quencher do not interact as efficiently as in the presence of the $\mathrm{C}_{4}$ substrate.

Thus, the fluorescence data correlate with the measured kinetic parameters since they both show a major difference between the $\mathrm{C}_{4}$ and the longer substrates (Fig. 7, Tables 1 and 2).

\section{DISCUSSION}

The mabA-inhA operon on the M. tuberculosis chromosome encodes two biochemically and structurally related enzymes. This study provides evidence that the MabA protein, like InhA, belongs to the mycobacterial FAS-II system, and that MabA catalyses the NADPHdependent reduction of $\beta$-ketoacyl derivatives, which corresponds to the second step of the elongation round. Thus, MabA and InhA carry out the two reduction steps of the elongation pathway. The inferred MabA model suggests the conservation of the quaternary structure of SDRs, in agreement with experimental data. Furthermore, the two regions of the substrate-binding pocket surrounding and facing the catalytic triad are very well conserved when compared to other KARs. Similar environments have been found in two related NADPHspecific keto reductases: the trihydroxynaphthalene reductase from Magnaporthe grisea (ThnR, PDB1YBV) and the tropinone reductase II from Datura stramonium (TR-II, PDB2AE2) (Andersson et al., 1996; Nakajima et al., 1999). These data lead us to propose similar orientations of the substrate keto function and catalytic mechanisms for the KARs (including MabA), ThnR and TR-II (Jörnvall et al., 1995; Thompson et al., 1997).

Both MabA and InhA have a large substrate-binding pocket, adequate to accommodate the long acyl chains elongated by FAS-II. The substrate specificity of MabA is also consistent with that of the M. tuberculosis KasIII (or $\mathrm{mtFabH}$ ) protein, believed to play the role of pivotal link between FAS-I and FAS-II systems (Choi et al., 2000). This specificity might partly reside in the relatively strong hydrophobicity of the MabA substratebinding pocket, particularly in the bottom part, which appears globally more hydrophobic than that of the other known KARs. It is noteworthy that, in the crystal structure of the ternary complex InhA-NAD ${ }^{+}-\mathrm{C}_{16}$ substrate, numerous hydrophobic residues interact with the long aliphatic chain of the substrate (Rozwarski et al., 1999). The steric hindrance and local conformational changes induced by a long acyl chain interacting with residues lining the MabA substrate-binding pocket may explain the difference in fluorescence behaviour between the $\mathrm{C}_{4}$ and $\mathrm{C}_{8}-\mathrm{C}_{16}$ substrates. Likewise, local rearrangements of the substrate-binding pocket of InhA and ThnR were observed upon binding of the substrate or an inhibitor (Rozwarski et al., 1999; Andersson et al., 1996).

The outbreak of resistant M. tuberculosis strains over the last 15 years has focused international attention on the need for new drug development. The structural and functional features of the MabA protein described here, especially its affinity for long-chain substrates, should allow the development of MabA-specific substrate analogues, as exemplified by thymidine monophosphate kinase of $M$. tuberculosis (Munier-Lehmann et al., 2001), which might lead to the design of novel antituberculous drugs.

\section{ACKNOWLEDGEMENTS}

We are grateful to Drs F. Laval and B. Montsarrat (IPBS, Toulouse) for MS analyses, and to L. Menu-Bouaouiche, V. Renaud, Dr P. Gouet (IPBS) and M. Cohen-Gonsaud (CBS, Montpellier) for their invaluable help. We thank Professor G. Lanéelle (IPBS) and Dr C. Royer (CBS) for helpful discussions. This work was supported in part by a doctoral fellowship (to H. Marrakchi) from the Fondation pour la Recherche Médicale, by a grant (QLK2-CT-2000-01761) from the European Community, and by the French Ministry of Research (Action Concertée Incitative: Molécules et Cibles Thérapeutiques).

\section{REFERENCES}

Altschul, S. F., Madden, T. L., Schäffer, A. A., Zhang, J., Zhang, Z., Miller, W. \& Lipman, D. J. (1997). Gapped BLAST and PSI-BLAST: a new generation of protein database search programs. Nucleic Acids Res 25, 3389-3402.

Andersson, A., Jordan, D., Schneider, G. \& Lindqvist, Y. (1996). Crystal structure of the ternary complex of 1,3,8-trihydroxynaphthalene reductase from Magnaporthe grisea with NADPH and an active-site inhibitor. Structure 4, 1161-1170.

Banerjee, A., Dubnau, E., Quémard, A., Balasubramanian, V., Um, K. S., Wilson, T., Collins, D., de Lisle, G. \& Jacobs, W. R., Jr (1994). $\operatorname{inh} A$, a gene encoding a target for isoniazid and ethionamide in Mycobacterium tuberculosis. Science 263, 227-230.

Banerjee, A., Sugantino, M., Sacchettini, J. C. \& Jacobs, W. R., Jr (1998). The mabA gene from the inhA operon of Mycobacterium tuberculosis encodes a 3-ketoacyl reductase that fails to confer isoniazid resistance. Microbiology 144, 2697-2704.

Beechem, J. M., Gratton, E., Ameloot, M. A., Knutson, J. R. \& Brand, L. (1991). The global analysis of fluorescence intensity and anisotropy decay data: second-generation theory and programs. In Fluorescence Spectroscopy, vol. 2, Principles and Techniques, pp. 241-301. Edited by J. R. Lakowicz. New York: Plenum.

Bloch, K. (1977). Control mechanisms for fatty acid synthesis in Mycobacterium smegmatis. Adv Enzymol 45, 1-84.

Choi, K. H., Kremer, L., Besra, G. S. \& Rock, C. O. (2000). Identification and substrate specificity of beta-ketoacyl (acyl carrier protein) synthase III ( $\mathrm{mtFabH}$ ) from Mycobacterium tuberculosis. J Biol Chem 275, 28201-28207.

Daffé, M. \& Draper, P. (1998). The envelope layers of mycobac- 
teria with reference to their pathogenicity. Adv Microb Physiol 39, 131-203.

Eisenberg, D., Luthy, R. \& Bowie, J. U. (1997). VERIFY3D: assessment of protein models with three-dimensional profiles. Methods Enzymol 277, 396-404.

Fisher, M., Kroon, J. T. M., Martindale, W., Stuitje, A. R., Slabas, A. R. \& Rafferty, J. B. (2000). The X-ray structure of Brassica napus beta-keto acyl carrier protein reductase and its implications for substrate binding and catalysis. Structure 8, 339-347.

Gouet, P., Courcelle, E., Stuart, D. I. \& Metoz, F. (1999). ESPript: analysis of multiple sequence alignments in PostScript. Bioinformatics 15, 305-308.

Jörnvall, H., Persson, B., Krook, M., Atrian, S., Gonzalez-Duarte, R., Jeffery, J. \& Ghosh, D. (1995). Short-chain dehydrogenases/ reductases (SDR). Biochemistry 34, 6003-6013.

Kraulis, P. J. (1991). MOLSCRIPT: a program to produce both detailed and schematic plots of protein structures. J Appl Cryst 24, 946-950.

Kremer, L., Douglas, J. D., Baulard, A. R. \& 9 other authors (2000). Thiolactomycin and related analogues as novel anti-mycobacterial agents targeting KasA and KasB condensing enzymes in Mycobacterium tuberculosis. J Biol Chem 275, 16857-16864.

Kremer, L., Nampoothiri, K. M., Lesjean \& 7 other authors (2001). Biochemical characterization of acyl carrier protein (AcpM) and malonyl-CoA:AcpM transacylase (mtFabD), two major components of Mycobacterium tuberculosis fatty acid synthase II. $J$ Biol Chem 276, 27967-27974.

Labesse, G. \& Mornon, J. P. (1998). A Tool for Incremental Threading Optimization (TITO) to help alignment and modelling of remote homologs. Bioinformatics 14, 206-211.

Labesse, G., Vidal-Cros, A., Chomilier, J., Gaudry, M. \& Mornon, J.-P. (1994). Structural comparisons lead to the definition of a new superfamily of $\mathrm{NAD}(\mathrm{P})(\mathrm{H})$-accepting oxidoreductases : the singledomain reductases/epimerases/dehydrogenases (the 'RED' family). Biochem J 304, 95-99.

Lakowicz, J. R. (1983). Protein fluorescence. In Principles of Fluorescence Spectroscopy, pp. 342-381. Edited by J. R. Lakowicz. New York: Plenum.

Laval, F., Lanéelle, M. A., Déon, C., Montsarrat, B. \& Daffé, M. (2001). Accurate molecular mass determination of mycolic acids by MALDI-TOF mass spectrometry. Anal Chem 73, 4537-4544.

Liu, J., Barry, C. E., 3rd, Besra, G. S. \& Nikaido, H. (1996). Mycolic acid structure determines the fluidity of the mycobacterial cell wall. J Biol Chem 271, 29545-29551.

Marrakchi, H., Lanéelle, G. \& Quémard, A. (2000). InhA, a target of the antituberculous drug isoniazid, is involved in a mycobacterial fatty acid elongation system, FAS-II. Microbiology 146, 289-296.

Mdluli, K., Slayden, R. A., Zhu, Y., Ramaswamy, S., Pan, X., Mead, D., Crane, D. D., Musser, J. M. \& Barry, C. E., 3rd (1998). Inhibition of a Mycobacterium tuberculosis beta-ketoacyl ACP synthase by isoniazid. Science 280, 1607-1610.

Munier-Lehmann, H., Chaffotte, A., Pochet, S. \& Labesse, G. (2001). Thymidylate kinase of Mycobacterium tuberculosis: a chimera sharing properties common to eukaryotic and bacterial enzymes. Protein Sci 10, 1195-1205.

Nakajima, K., Kato, H., Oda, J., Yamada, Y. \& Hashimoto, T. (1999). Site-directed mutagenesis of putative substrate-binding residues reveals a mechanism controlling the different stereospecificities of two tropinone reductases. J Biol Chem 274, 16563-16568.

Odriozola, J. M., Ramos, J. A. \& Bloch, K. (1977). Fatty acid synthetase activity in Mycobacterium smegmatis. Characterization of the acyl carrier protein-dependent elongating system. Biochim Biophys Acta 488, 207-217.

Quémard, A., Lacave, C. \& Lanéelle, G. (1991). Isoniazid inhibition of mycolic acid synthesis by cell free extracts of sensitive and resistant strains of Mycobacterium aurum. Antimicrob Agents Chemother 35, 1035-1039.

Quémard, A., Sacchettini, J. C., Dessen, A., Vilchèze, C., Bittman, R., Jacobs, W. R., Jr \& Blanchard, J. S. (1995). Enzymatic characterization of the target for isoniazid in Mycobacterium tuberculosis. Biochemistry 34, 8235-8241.

Quémard, A., Dessen, A., Sugantino, M., Jacobs, W. R., Jr, Sacchettini, J. C. \& Blanchard, J. S. (1996). Binding of catalaseperoxidase-activated isoniazid to wild-type and mutant $\mathrm{Myco-}$ bacterium tuberculosis enoyl-ACP reductases. I Am Chem Soc 118, 1561-1562.

Rafferty, J. B., Simon, J. W., Baldock, C., Artymiuk, P. J., Baker, P. J., Stuitje, A. R., Slabas, A. R. \& Rice, D. W. (1995). Common themes in redox chemistry emerge from the X-ray structure of oilseed rape (Brassica napus) enoyl acyl carrier protein reductase. Structure 3, 927-938.

Rozwarski, D. A., Grant, G. A., Barton, D. H. R., Jacobs, W. R., Jr \& Sacchettini, J. C. (1998). Modification of the NADH of the isoniazid target (InhA) from Mycobacterium tuberculosis. Science 279, 98-102.

Rozwarski, D. A., Vilchèze, C., Sugantino, M., Bittman, R. \& Sacchettini, J. C. (1999). Crystal structure of the Mycobacterium tuberculosis enoyl-ACP reductase, InhA, in complex with $\mathrm{NAD}^{+}$ and a $\mathrm{C}_{16}$ fatty acyl substrate. J Biol Chem 274, 15582-15589.

Sali, A. \& Blundell, T. L. (1993). Comparative protein modelling by satisfaction of spatial restraints. J Mol Biol 234, 779-815.

Sheldon, P. S., Kekwick, R. G. O., Sidebottom, C., Smith, C. G. \& Slabas, A. R. (1990). 3-Oxoacyl-(acyl-carrier protein) reductase from avocado (Persea americana) fruit mesocarp. Biochem J 271, 713-720.

Sheldon, P. S., Kekwick, R. G., Smith, C. G., Sidebottom, C. \& Slabas, A. R. (1992). 3-Oxoacyl-[ACP] reductase from oilseed rape (Brassica napus). Biochim Biophys Acta 1120, 151-159.

Shimakata, T. \& Stumpf, P. K. (1982). Purification and characterization of $\beta$-ketoacyl-[acyl-carrier-protein] reductase, of $\beta$ hydroxyacyl-[acyl-carrier-protein] dehydrase, and enoyl-[acylcarrier-protein] reductase from Spinacea oleracea leaves. Arch Biochem Biophys 218, 77-91.

Sippl, M. J. (1993). Recognition of errors in three-dimensional structures of proteins. Proteins 17, 355-362.

Takayama, K., Wang, L. \& David, H. L. (1972). Effect of isoniazid on the in vivo mycolic acid synthesis, cell growth, and viability of Mycobacterium tuberculosis. Antimicrob Agents Chemother 2, 29-35.

Thompson, J. D., Higgins, D. G. \& Gibson, T. J. (1994). Clustal w : improving the sensitivity of progressive multiple sequence alignment through sequence weighting, position-specific gap penalties and weight matrix choice. Nucleic Acids Res 22, 4673-4680.

Thompson, J. E., Basarab, G. S., Andersson, A., Lindqvist, Y. \& Jordan, D. B. (1997). Trihydroxynaphthalene reductase from Magnaporthe grisea: realization of an active center inhibitor and elucidation of the kinetic mechanism. Biochemistry 36, 18521860.

Vagelos, P. R. \& Alberts, A. W. (1960). Chemical synthesis of $\beta$ ketooctanoyl coenzyme A. Anal Biochem 1, 8-16.

Vilchèze, C., Morbidoni, H. R., Weisbrod, T. R., Iwamoto, H., Sacchettini, J. C. \& Jacobs, W. R., Jr (2000). Inactivation of the 
inhA-encoded fatty acid synthase II (FASII) enoyl-acyl carrier protein reductase induces accumulation of the FASI end products and cell lysis of Mycobacterium smegmatis. J Bacteriol 182, 4059-4067.

Zabinski, R. F. \& Blanchard, J. S. (1997). The requirement for manganese and oxygen in the isoniazid-dependent inactivation of
Mycobacterium tuberculosis enoyl reductase. J Am Chem Soc 119, 2331-2332.

Received 9 November 2001; revised 28 November 2001; accepted 29 November 2001. 